\title{
Recursos naturales en la región de los valles, Jalisco. \\ Natural resources in the region of the valley, Jalisco
}

\author{
Lilia Francisca Sánchez Moreno \\ Universidad de Guadalajara \\ lilia.sanchez@profesores.valles.udg.mx
}

\section{Resumen}

La Región Valles se encuentra en los límites de las provincias fisiográficas IV Sierra Madre occidental y X Eje Neovolcánico correspondiendo los sistemas de topoformas predominantes a sierras, lomeríos y mesetas según la Comisión Estatal de Agua, Jalisco (CEA, Jal). Esta Región Valles se encuentra en el centro occidente del Estado y colinda con las regiones 01 Norte, 10 Sierra Occidental, 12 Centro, 06 Sur y 07 Sierra de Amula, así como con el Estado de Nayarit. Tiene una superficie de $5,851 \mathrm{~km}^{2}$, lo cual representa 7.3 por ciento del total Estatal, lo cual ubica a Valles como una área geográfica media, con relación al resto de las regiones del Estado de Jalisco.

Esta región tiene una gran riqueza en cuanto a Recursos Naturales se refiere, ya que cuenta con la recién declarada Área Estatal de Protección Hidrológica, "Sierra del Águila" el 18 de febrero del 2010, Presa de la Vega declarada sitio Ramsar en el mismo año y tiene uno de los recursos hídricos más importantes de Jalisco, Cuenca del Río Ameca.

De acuerdo con la Comisión Nacional del Agua (CONAGUA), la región Valles es una superficie donde la capacidad de los mantos acuíferos permite la extracción para cualquier tipo de uso, encontrando dos zonas geohidrológicas donde se realizan extracciones, que son la de Tequila (RH12) y la de Ameca (RH14).

Palabras clave: Recursos Naturales, biodiversidad, conservación, Áreas Naturales Protegidas, Río Ameca, flora, fauna. 


\section{Abstract}

The Valles region is on the edge of the physiographic provinces western Sierra Madre IV and $\mathrm{X}$ Neovolcanic corresponding systems predominant topography in the reserve to mountains, hills and plateaus according to state water commission, Jalisco (CEA, Jal). This Region Valles is located in central western borders of the state and the regions 01 North 10 West Sierra 12 Center 06 South and 07 Sierra de Amula as well as with the State of Nayarit. It has an area of $5,851 \mathrm{~km} 2$, which represents 7.3 percent of the state total, which places Valles as an average geographic area, relative to other regions of the State of Jalisco.

This region is rich in natural resources are concerned, as it has just declared State Area Hydrological Protection, "Sierra del Aguila" the February 18, 2010, Presa de la Vega declared a Ramsar site in the same year and he has one of the most important water resources in Jalisco, Ameca River Basin.

According to the National Water Commission (CONAGUA), the Valles region is an area where the capacity of aquifers allows extraction for any type of use, by finding two geohydrological areas where withdrawals are made, which are Tequila (RH12) and Ameca (RH14).

Key words: Natural resources, biodiversity conservation, protected areas, Rio Ameca, flora, fauna.

Fecha Recepción: Septiembre 2014 Fecha Aceptación: Noviembre 2014

\section{INTRODUCCIÓN}

Por su ubicación geográfica México presenta una extraordinaria diversidad biológica y de ecosistemas. Con apenas el $1.47 \%$ de la superficie terrestre del planeta, ocupa el cuarto lugar entre los países considerados con megadiversidad biológica y posee cerca del 10\% del total de especies conocidas con un gran número de endemismos. 
En el ámbito mundial, México ocupa el quinto lugar con respecto al número de especies de plantas y el primer lugar en especies de pinos.

Jalisco es uno de los estados de mayor complejidad ecológica e importancia para la conservación de la biodiversidad nacional. Con poco más de 4\% de la superficie terrestre del país, se encuentra entre los primeros seis lugares de los estados prioritarios para su conservación, lugar que corresponde a su gran riqueza biológica.

Es una región privilegiada debido a que tiene una gran riqueza en cuanto a Recursos Naturales se refiere, ya que cuenta con la recién declarada Área Estatal de Protección Hidrológica, "Sierra del Águila" el 18 de febrero del 2010, Presa de la Vega declarada sitio Ramsar en el mismo año y tiene uno de los recursos hídricos más importantes de Jalisco, Cuenca del Río Ameca.

Está conformada por 14 Municipios que son: Ahualulco de Mercado, Amatitán, Ameca, San Juanito Escobedo, El Arenal, Cocula, Etzatlan, Hostotipaquillo, Magdalena, San Marcos, San Martín Hidalgo, Tala, Tequila y Teuchitlán. La sede de esta región es el municipio de Ameca.

Esta región es de gran importancia en el estado, ya que se llevan a cabo actividades de agricultura, ganadería, explotación forestal, cañera principalmente en el Municipio de Tala donde se encuentra el ingenio azucarero más grande del Estado, apicultura y la industria tequilera; se destaca por las artesanías y la joyería, Magdalena por la Minería, sobre todo la explotación de las Minas de ópalo. Además de la zona arqueológica llamada Guachimontones que es muy rara ya que son edificaciones en círculos concentricos en Teuchitlan.

Cada uno de estos municipios cuenta con hermosos poblados cada uno de ellos con particularidades especiales, en algunos encontramos "brazos del río Ameca" que atraviesan el poblado y en otros como por ejemplo Teuchitlan con su río del mismo nombre que desemboca en la Presa de la Vega y de ahí surge con su majestuosidad el río Ameca, del cual riegan los cultivos existentes y abastece del vital líquido a los pobladores, observamos el paisaje en estos vasos lacustres con su flora y fauna característica, en algunos sitios 
podemos observar a la Sierra Madre Occidental, como por ejemplo en Ameca observamos el Cerro Grande de Ameca que forma parte del Área Estatal de Protección Hidrológica Sierra del Águila, a este Cerro también se le conoce como Cerro Cuauhtépetl o Cerro del Águila con su flora particular.

Para hablar un poco más del río Ameca es la más importante corriente fluvial no solo del municipio sino de la parte occidental de nuestro estado, que nace en la inmediaciones del poblado de La primavera, en el municipio de Zapopan; luego recibe los caudales de los ríos Teuchitlán, Tala, Cuisillos, Ahualulco, Cocula, San Martín Hidalgo, Atenguillo y Mascota; para luego desembocar en la Bahía de Banderas a inmediaciones de la ciudad de Puerto Vallarta. Es importante hacer notar que por medio de canales construidos exprofeso el río Ameca recibe las aguas de la Laguna de San Juanito y La Magdalena. En su curso el río Ameca atraviesa los municipios de Zapopan, Tala, Teuchitlán, San Martín Hidalgo, Ameca, Guachinango, Mascota, San Sebastián del Oeste y Vallarta. Tiene una extensión de 260 Kms. de largo, un escurrimiento anual de $2,500 \mathrm{~m}^{3}$ de agua y una cuenca de $14,000 \mathrm{Kms}^{2}$. Sobre el cauce del río Ameca se construyó la presa de la Vega, cuyo almacenamiento acuífero es de 44000 $000 \mathrm{de}^{3}$ anuales y una capacidad de irrigación para 9,000 hectáreas. (Filemón Gutiérrez Ramírez, 1982).

Doce municipios han sido evaluados como sobreexplotados en este rubro, siendo Etzatlan el que está dentro de esta categoría, otro está catalogado como en equilibrio (Ahualulco de Mercado) y tan solo uno como sobreexplotado, siendo éste Ameca, lo cual sin duda se debe a la presencia del distrito de riego, y de la extracción excesiva de este elemental líquido. Sin embargo, un tanto al margen de este caso problemático (el del municipio de Ameca), en parte atendido mediante vedas flexibles, más bien destaca un gran potencial que queda por explotarse, facilitando no solo las actividades agropecuarias, sino también industriales y de servicios en los centros urbanos. 


\section{CARACTERISTICAS GENERALES}

Orografía: existe una gran variedad de terrenos desde serranía, lomeríos y valles. En la región se encuentra el cerro de Ameca, que es uno de los más altos de nuestro valle y que viene siendo una ramificación de la Sierra Madre Occidental, en él se inicia la serranía de Ameca, y que va a terminar en Ahuacatlan, Nayarit y tiene una extensión de cincuenta y ocho kilómetros.

Hidrografía: a todo lo largo pasa el río Ameca, que después del río Santiago, es uno de los más importantes del sistema hidrográfico del estado de Jalisco. (Gutiérrez Ramírez 1982)

Desde un enfoque fisiográfico la zona pertenece a la provincia de la Faja Volcánica Mexicana, cerca de los límites con la Sierra Madre Occidental, siendo parte de la porción sur del Bloque Jalisco, con una altitud de $1240 \mathrm{msnm}$.

\section{FLORA EN LA REGIÓN}

La determinación de la flora localizada en el área de estudio al realizar las colectas e identificación respectiva, corresponde a los tipos de vegetación según la clasificación del Botánico Jerzy Rzedowski (1978), misma en la que se sustentan los listados florísticos del Instituto Nacional de Estadística y Geografía (INEGI) y de la Comisión Nacional Forestal (CONAFOR).

La codificación asignada a cada tipo de vegetación, será la que se menciona a continuación, misma que aparece en el listado de las especies que se colectaron y observaron en el sitio de estudio.

Bosque de Encino (BE).

Bosque de Encino-Pino (BE-P)

Bosque de Galería (BG)

Selva Baja Caducifolia (SBC).

Otros (Árboles frutales) 


\section{Bosque de Encino (BE).}

Comunidad vegetal formada por diferentes especies de encinos o robles del género Quercus. Estos bosques generalmente se encuentran como una transición entre los bosques de coníferas y las selvas, pueden alcanzar desde los 4 hasta los $30 \mathrm{~m}$. de altura en lugares más o menos abiertos o muy densos. (CONAFOR 2014).

\section{Bosque de Encino-Pino (BE-P.)}

Vegetación arbórea formada por la dominancia de encinos (Quercus spp), sobre los pinos (Pinus spp.). Los Encinos se desarrollan principalmente en áreas de mayor importancia forestal, en los límites altitudinales inferiores de los bosques de pino-encino. Estas comunidades muestran menor porte y altura que aquellos en donde domina el pino sobre el encino. A este tipo de vegetación se le considera como ecosistemas templado-frío que abarca los tipos de vegetación cuya distribución corresponde en general a la ubicación de las serranías más importantes de México (CONAFOR 2014).

\section{BOSQUE DE GALERÍA (BG).}

Comunidad arbórea que se encuentra en los márgenes de los ríos o arroyos con cauce de agua permanente o intermitente, es decir donde existan condiciones de humedad favorable. La localización de este tipo de vegetación en México en general, es un tanto compleja dado la ubicación dentro de otros sistemas vegetativos, lo que conduce a que muchas veces la diferenciación específica de los componentes hace referencias a otras comunidades principalmente con la de la Selva Baja Caducifolia y con los Bosques de Encino, EncinoPino. Una característica fisionómica particular y que alude su nombre es que en algunos casos, esta vegetación se presenta de forma exuberante, los árboles forman una especie de galería a lo largo de la corriente acuática. (CONAFOR 2014). 


\section{SELVA BAJA CADUCIFOLIA (SBC).}

Son comunidades formadas por vegetación arbórea de origen meridional (Neotropical), generalmente de climas cálido húmedo, subhúmedo y semiseco. Están compuestas por la mezcla de un gran número de especies, muchas de las cuales presentan contrafuertes o aletones. En algunas áreas observadas presentan bejucos, lianas y plantas epífitas, frecuentemente con arbustivas espinosos entre los dominantes, como los huizaches. (CONAFOR 2014).

\section{OTROS.}

Existe una variedad de árboles frutales como son: mango, guayaba, guamúchil y pitayo.

\section{FAUNA}

En la bibliografía consultada y entrevistas con biólogos que realizaron trabajo de campo se tiene que, se estudiaron todos los grupos faunísticos y se consultó la NOM-059SEMARNAT-2001, con el fin de identificar la posible presencia de especies bajo algún tipo de Categoría, de acuerdo los listados de la norma oficial mexicana NOM-059SEMARNAT-2001 misma que refiere a las especies y subespecies de flora y fauna terrestres y acuáticas en peligro de extinción, amenazadas, raras, y las sujetas a protección especial, y dentro de las categorías endémicas a la república mexicana y aguas de jurisdicción federal, cabe hacer mención que ninguna de las especies observadas se encuentra dentro de la norma oficial mexicana NOM-059-SEMARNAT-2001.

Aves.

El listado de aves se realiza principalmente a través de observación, mediante recorridos por veredas, claros, arroyos y zonas arboladas. Para su posterior identificación, se utilizaron las guías de campo: Aves de México, de Peterson y Chalif (1989). Así también, como una revisión de literatura con el fin de conocer las especies de aves (residentes y migratorias) observadas; y la consulta a los pobladores sobre la presencia de ciertas aves. 


\section{Mamíferos.}

El registro de los mamíferos, se lleva a cabo mediante recorridos por cañadas, arroyos y veredas, para la observación de huellas, excretas, rastros e incluso a algunas especies atropelladas en caminos. La identificación de las especies se realiza con el apoyo del libro "Rastros de los mamíferos silvestres de México" (Aranda Sánchez, 1981). Se consultó la literatura y a los pobladores quienes proporcionaron información referente a algunas especies comunes en la zona.

Reptiles y anfibios.

El registro de las especies de estos dos grupos zoológicos se realiza a través de caminatas por los sitios rocosos, zonas boscosas, márgenes de los arroyos y cuerpos de agua. La identificación de las especies (incluyendo especies atropelladas) fue mediante la observación, detección de rastros, refugios. Para las especies con hábitos nocturnos y crepusculares (puesto que algunos anfibios y serpientes se alimentan en esos horarios), se hicieron caminatas también por veredas. Además de realizar la consulta a los pobladores del lugar sobre los ejemplares que ellos han visualizado. Posteriormente, se examinó la literatura para corroborar las especies mencionadas.

Como ejemplo de algunas de ellas podemos mencionar las siguientes especies, aves: carpintero desértico, Carpintero arlequín, Urraca copetona, Papamoscas cejiamarillo, Correcaminos, Mosquero cardenalito, Cardenal rojo, Güilota, Paloma alas blancas, Golondrina tijereta, Cenzontle, Gallaretas, Garzas. Reptiles y anfibios: Tortuga casquito Iguanas, Lagarto, Cascabel. Mamíferos: Armadillo Jabalí, Venado, Zorrillo, Leoncillo

\section{RÍO AMECA}

De acuerdo con la Comisión Nacional del Agua (CONAGUA), la región Valles es una superficie donde la capacidad de los mantos acuíferos permite la extracción para cualquier 
tipo de uso, encontrando dos zonas geohidrológicas donde se realizan extracciones, que son la de Tequila (RH12) y la de Ameca (RH14).

Es importante hacer notar que por medio de canales construidos ex profeso el río Ameca recibe las aguas de la Laguna de San Juanito y La Magdalena. En su curso el río Ameca atraviesa los municipios de Zapopan, Tala, Teuchitlán, San Martín Hidalgo, Ameca, Guachinango, Mascota, San Sebastián del Oeste y Vallarta. Tiene una extensión de 260 $\mathrm{Km}$. de largo, un escurrimiento anual de $2,500 \mathrm{~m}^{3}$ de agua y una cuenca de $14,000 \mathrm{Kms}^{2}$. Sobre el cauce del río Ameca se construyó la presa de la Vega, cuyo almacenamiento acuífero es de 44000 $000 \mathrm{de}^{3}$ anuales y una capacidad de irrigación para 9,000 hectáreas. (Filemón Gutiérrez Ramírez, 1982).

En la época de 1910 se sabe que el llamado río de Ameca adquiere una gran importancia de carácter social, de recreación ya que se efectuaban competencias de regatas, de natación, de proveedor de alimento ya que era muy característico la especie de pescado bagre Pimelodus labrosus, fue la ducha para la gran mayoría de la población y también el lugar común donde las mujeres de aquel tiempo concurrían a lavar las ropas de sus familias. Sabemos que en aquel tiempo, este río, era caudaloso, al grado de que los meses de sequía como marzo, abril y mayo, siempre estaban cubiertos de agua, los nueve arcos de que se componía el puente en la ciudad de Ameca. Para reforzar ya de por si aquel caudaloso río, tendré que decir que frente a la Hacienda de San Francisco, se levantaban año por año, una presa para retener las aguas del mencionado río, con el objeto de hacer regadíos en los terrenos de la mencionada propiedad. Se ofreciera un atrayente paseo. Y así lo era en efecto, pues era uno de los sitios que se consideraban como uno de los paseos con que contaban las familias que radicaban en aquel entonces en la cuidad de Ameca, pues estas, frecuentemente hacían su paseo al lado del puente donde se sentaban a uno y otro lado de los acantilados de dicho, para mí, hermoso puente. También había canoas con remos, y además había pequeñas lanchitas de gasolina, que circulaban desde el puente, río arriba, hasta la Hacienda La Esperanza, y se daban gusto los jóvenes de ese tiempo en hacer prácticas de remo y haber quien llegaba en primer lugar a la terminal que se hacía en la Hacienda la Esperanza. (J. Concepción Díaz Navarro, 2005) 
El río Ameca nace en la Sierra de la Primavera en el municipio de Zapopan Jalisco y se le da el nombre de rio Caliente debido a que es una zona de aguas termales. Originalmente se ha identificado una extensa zona geohidrológica desde el nacimiento del río Ameca hasta los límites con el estado de Nayarit, aguas debajo de Ameca.

El agua subterránea sigue las direcciones y sentidos de las principales corrientes superficiales, especialmente las de los ríos Salado y Ameca junto con sus afluentes principales. El flujo subterráneo en la zona de Magdalena es concéntrico al valle principal.

Los manantiales que se identifican en la zona de Ameca se localizan en pie de monte y algunos de ellos, al oriente de este valle presentan ciertas características de termalismo, aunque con una temperatura ligeramente superior a la media y en general de poco gasto. (CONAGUA 2014).

Extracción de aguas subterráneas. La zona de Ameca cuenta con la mayor extensión superficial plana y esto sugiere que las aguas subterráneas son las más explotadas, sin embargo dos factores contribuyen a disminuir el potencial de usos más consuntivos como los agropecuarios, por una parte la existencia de la zona de riego de aguas superficiales y por la otra, que el espesor de materiales granulares de menor permeabilidad, es menor que en las otras zonas.

Para la zona de Ameca y su zona de recarga, en un área de $1391 \mathrm{~km}^{2}$, llueve un volumen de 1336.61 $\mathrm{Mm}^{3} / \mathrm{año}$, se evapotranspiran alrededor de $1003.38 \mathrm{Mm}^{3} / \mathrm{a}$, y escurren 144.418 , por lo que la infiltración resulta de $188.81 \mathrm{Mm}^{3}$ anuales.( CONAGUA 2014).

El agua es ha sido y será un recurso muy importante en la historia del hombre y sus beneficios han sido significativos desde el descubrimiento de la agricultura, por lo que se puso de manifiesto la necesidad de contar con suficientes reservas de agua, para cubrir esa y muchas otras necesidades, tales como: La ganadería, mantener las funciones de los organismos vivos, la higiene personal y su empleo domestico, la generación de energía, las 
actividades productivas como la acuicultura y pesca, la regulación del clima, la generación de oxigeno, además de servir como belleza escénica, para realizar actividades deportivas y de salud como en la hidroterapia, entre otras. (Gobierno de Jalisco, 2015).

El agua tiene un doble valor para la ecología, primero porque es un elemento de un ecosistema y es consecuentemente un activo social; segundo es un generador de ecosistemas. Por ser cuestiones muy importantes a considerar, cuando se trata de llevar a cabo aprovechamientos de agua, la conservación de la especies y de los ecosistemas afectados no podemos olvidar la función que realiza el agua cuando fluye de modo variable, desde las cabeceras de los ríos hasta el mar, puesto que moviliza y distribuye elementos químicos tan importantes para la vida. La función ecológica en sus dos vertientes fundamentales, a) mantenimiento de los ecosistemas que le son propios, b) el agua es el vehículo de transporte de nutrientes, sedimentos y vida, es un bien común que debe conciliarse con las actividades humanas sobre la tierra. (L. F. Sánchez Moreno, et al, 2007).

Afortunadamente, el agua dulce disponible se nutre de un generoso suministro que continuamente se recoge, purifica, recicla y distribuye en el ciclo hidrológico impulsado por la energía solar, tanto tiempo como no la sobrecarguemos con residuos no degradables o de lenta degradación o retiremos los suministros de aguas subterráneas más rápidamente de lo que tardan en rellenarse. Desgraciadamente hacemos ambas cosas. (G. Tyller Miller, Jr., 2002).

Es imprescindible revisar los criterios de asignación del agua, especialmente en lo que respecta a las actividades económicas. La incidencia negativa que los nuevos criterios pudieran tener sobre algunos regadíos (hay distintas agriculturas y distintos agricultores), debe ser corregida considerando la función social que es inherente a la mayor parte de esos regadíos. La subvención de los costes del agua es contraproducente con la asignación eficaz del propio recurso. (L. F. Sánchez Moreno, et al, 2007). 


\section{CONCLUSIONES.}

Esta región de Jalisco es rica en Recursos Naturales ya que tiene en su territorio al Río Ameca, "Sierra del Águila" Área Estatal de Protección Hidrológica, Presa de la Vega como Sitio Ramsar, que en conjunto sirven de hábitat para una gran cantidad de fauna y con una gran variedad de flora que va desde el bosque de encino-pino hasta las praderas y matorrales; se considera uno de los corredores ecológicos más importantes de Jalisco y de México.

De una manera general conserva su flora lo que provee de servicios ambientales a la comunidad y en consecuencia a la región de los Valles, además de otro elemento de suma importancia como lo es el río Ameca, que conjuntamente con la serranía cumplen con la función de un ecosistema de características especiales y son reguladores climáticos.

\section{PRESENTACIÓN GRÁFICA}

\section{Región Valles}

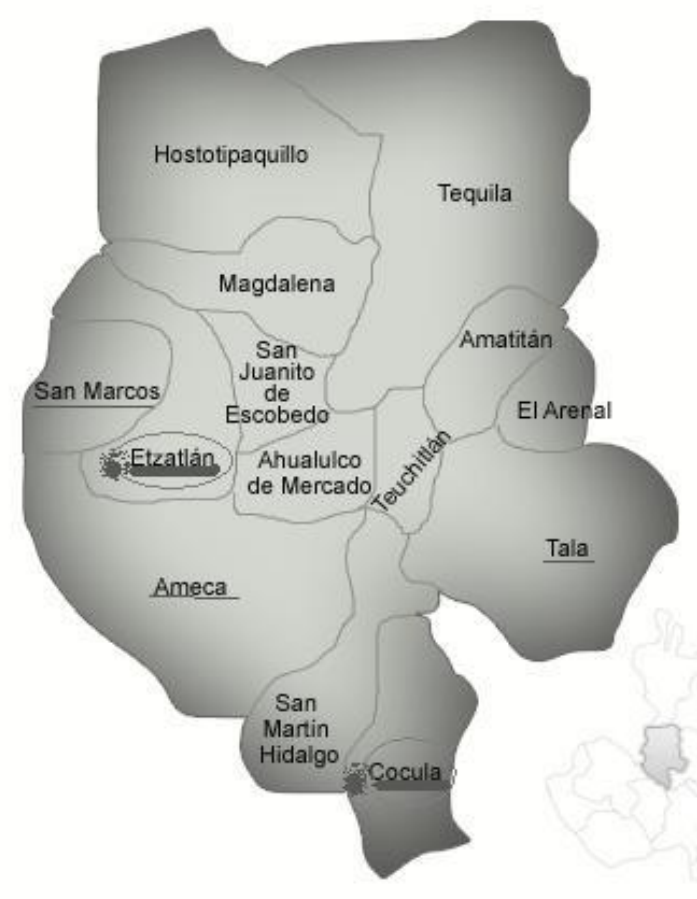


Mapa 1. Región Valles, Jalisco México.jalisco.gob.mx/es/jalsico/municipios

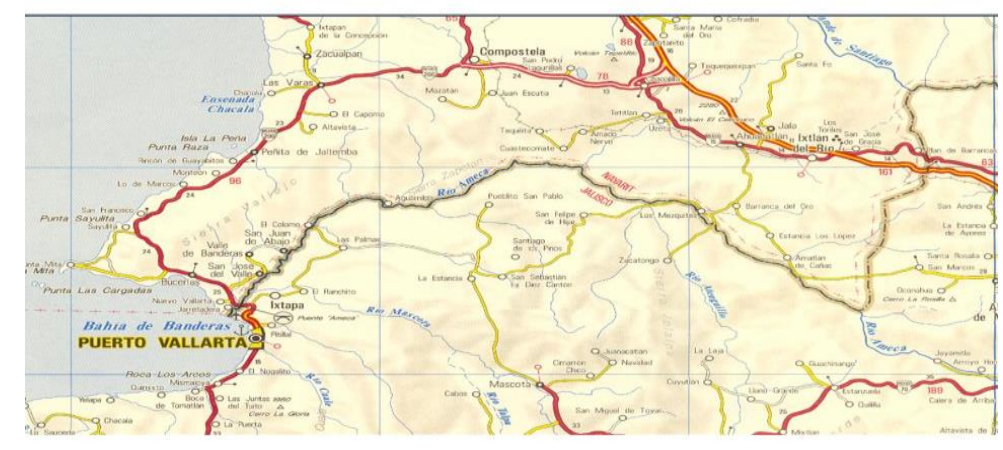

Mapa 2. Trayecto Río Ameca de Jayamitla hasta Bahia de Banderas, Puerto Vallarta Jalisco. Google Maps enero 2015

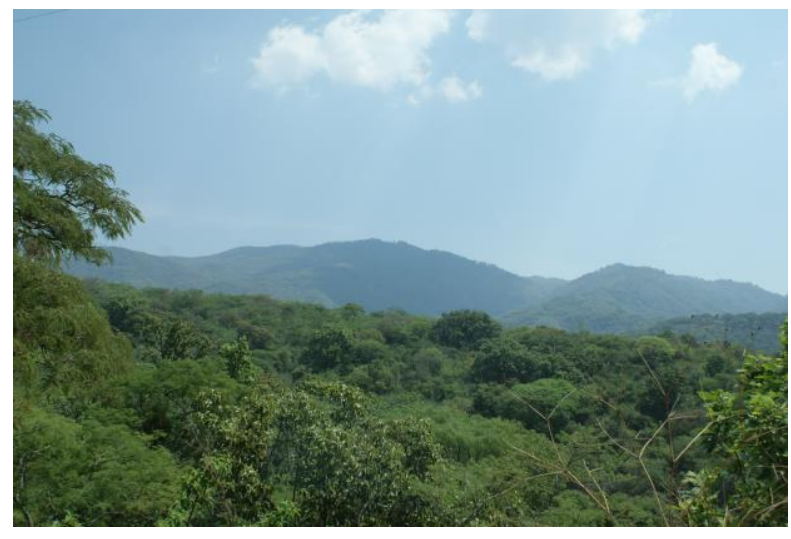

Fotografía 1. Bosque encino. De la colección Sánchez Moreno 2014. 


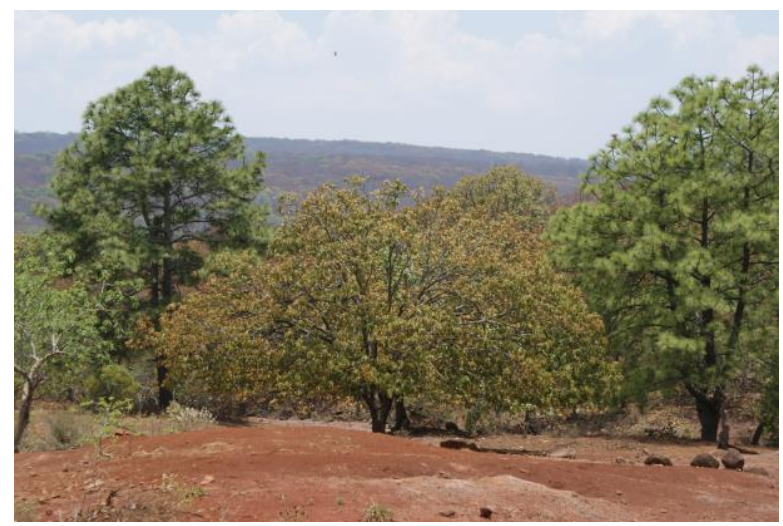

Fotografía 2. Bosque encino-pino. De la colección Sánchez Moreno 2014.

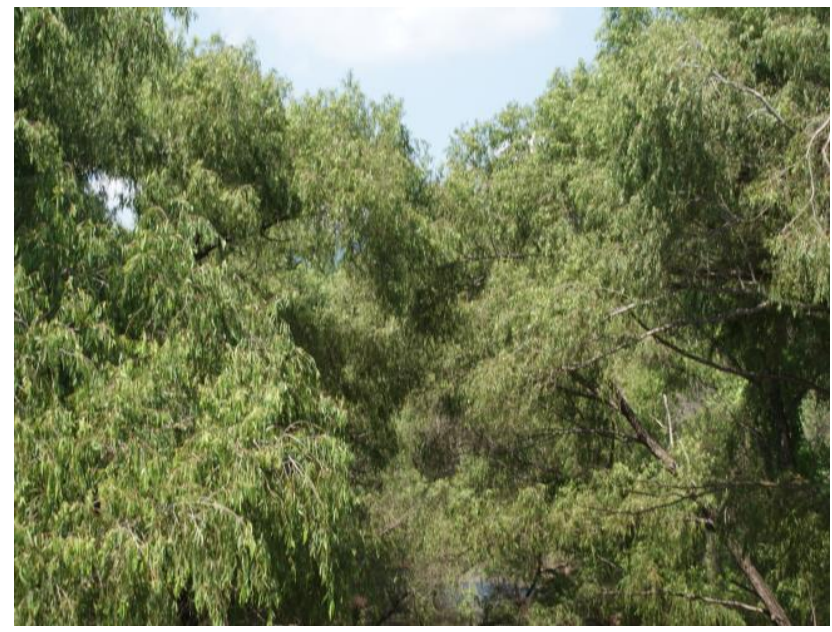

Fotografía 3. Bosque de galería. De la colección Sánchez Moreno 2014. 


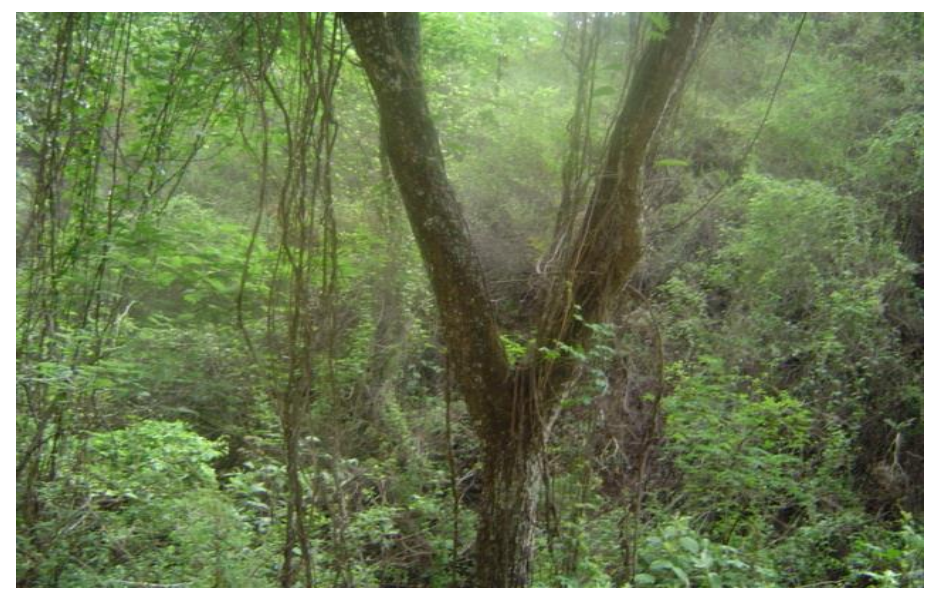

Fotografía 4. Selva baja caducifolia. De la colección Sánchez Moreno 2014.

\section{Bibliografía}

Aranda Sánchez, 1981.Rastros de los mamíferos silvestres de México,

Filemón Gutiérrez Ramírez, 1982, AMECATL, MECATE O CORDON DE AGUA, $1^{\text {a }}$. Edición, Suarez-muñoz EDICIONES,

Gobierno de Jalisco, Secretaria del Medio Ambiente para el Desarrollo Sustentable, 2007, Guía del maestro, calendario ambiental, segunda edición, Cisneros Rivera Impresores,

SEMARNAT-2001.

Peterson y Chalif,1989., Aves de México.,

G. Tyller Miller, Jr. 2002, Ciencia ambiental, Preservemos la Tierra, 5a . Edición, International Thomson Editores,

G. TYLLER MILLER, JR.1994 ECOLOGÍA Y MEDIO AMBIENTE, Introducción a, la Ciencia Ambiental, el desarrollo sustentable y la conciencia de conservación del Planeta Tierra, Grupo Editorial Iberoamérica,.

J. Concepción Díaz Navarro, 2005, AMECA Y SUS COSTUMBRES EN 1910, Segunda edición, Velma Editores,

Lilia Francisca Sánchez Moreno, et al, 2007.CRITERIOS DE CARÁCTER SOCIAL Y ACCIÓN TECNOLÓGICA SOBRE EL TRATAMIENTO Y DEPURACIÓN DEL AGUA, Primera Edición, Editorial Amate. 
Programa para las Naciones Unidas para el Medio Ambiente, 2002, Perspectivas del Medio Ambiente Mundial GEO-3, Primera edición, Ediciones Mundi-Prensa,

\section{Fotografías.}

De la colección Sánchez Moreno 2013-2014. 